\title{
Pain in Burning Mouth Syndrome- Neuropathogenic Hypothesis
}

ISSN: 2637-7764

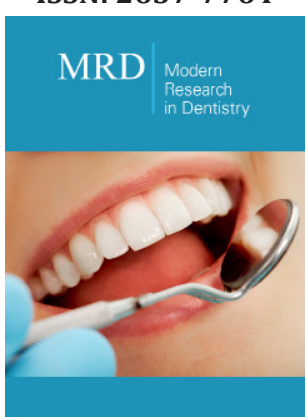

*Corresponding author: Bogdan-Ioan Coculescu, Faculty of Medicine, Titu Maiorescu University, 67A Gheorghe Petrașcu, Bucharest, Romania

Submission: 盟 May 17, 2021

Published: 湢June 01, 2021

Volume 6 - Issue 3

How to cite this article: Elena Claudia Coculescu, Gheorghe Manole, Carmen Nicolae, Bogdan-Ioan Coculescu. Pain in Burning Mouth Syndrome-Neuropathogenic Hypothesis. Mod Res Dent. 6(3) MRD. 000639. 2021.

DOI:_10.31031/MRD.2021.06.000639

Copyright@ Bogdan-Ioan Coculescu, This article is distributed under the terms of the Creative Commons Attribution 4.0 International License, which permits unrestricted use and redistribution provided that the original author and source are credited.

\author{
Elena Claudia Coculescu ${ }^{1}$, Gheorghe Manole ${ }^{2}$, Carmen Nicolae ${ }^{1}$ and Bogdan- \\ Ioan Coculescu ${ }^{3,4 *}$ \\ ${ }^{1}$ Faculty of Dental Medicine, Carol Davila University of Medicine and Pharmacy, Bucharest, \\ Romania \\ ${ }^{2}$ Faculty of General Nursing, Bioterra University, Bucharest, Romania \\ ${ }^{3}$ Cantacuzino National Medico-Military Institute for Research and Development, Bucharest, \\ Romania \\ ${ }^{4}$ Faculty of Medicine, Titu Maiorescu University, Bucharest, Romania
}

\begin{abstract}
Burning mouth syndrome (BMS) in its primary clinical form has controversial etiopathogenesis, which explains the frequency of therapeutic failures. On the other hand, the characteristics of the oral pain that allow it to be identified as a particular form of pain are an impediment to its classification in a certain category: through functional lesion/disruption of receptors, of neuropathy or cortical type. Based on their professional experience and the scientific data from the specialized medical literature, the authors raise the hypothesis of the existence of a neuronal irrigation deficiency, manifested both at the conductive sensory fibers of the influx and, preferably, in the sensory-sensorial cortex for pain and taste, or their association. According to the hypothesis, neural irrigation is responsible for installing a shortage of energy production and use, as well as the local synthesis of excess reactive oxygen species that engages the disruption of conduction of the thermoalgesic nervous influx generated in the oral/lingual mucosa.
\end{abstract}

Keywords: Oxidative stress; Neuropathic pain; Cortical pain

Abbreviation: BMS: Burning Mouth Syndrome

\section{Introduction}

Since the definition of neuropathic pain in 1994 by the International Association for the Study of Pain was of a general nature, in 2008, the Neuropathic Pain Study Working Group within the same scientific forum pain has been redefined as "the pain as a direct consequence of the injury or of affecting the somatosensory system" [1,2]. The characteristic feature of the primary form of BMS to develop paroxystically, spontaneously, in the absence of an inducing stimulus, or to exacerbate very varied stimuli, but also to associate abnormal sensations, allows the pain to be classified as neuropathic. As it deteriorates the quality of life, with socio-professional consequences, BMS's symptomatology justifies the interest of its etiopathogenesis study, which, if identified, would also provide effective management tools for this condition [3-6]. Starting from our finding that the BMS sensitivity disorder is localized to a particular neuro-anatomical area, we argue that the symptomatology of the syndrome is the most likely expression of the development of predominantly functional disturbances at the peripheral tract of the cranial nerves. It consists of the dendritic extensions, respectively of the axon of the neuron I located in the nerve attached to the cranial nerves, which it forms. In our view, dysfunctionality is predominantly axonal, as a consequence of the development of microangiosclerosis in the vascular plexus whose tract accompanies the nerve, from which two independent functional sources are detached: the extrinsic, epineural and intrinsic endoneural system [4-9].

\section{Material and Methods}

In an attempt to explain the pathogenesis of pain syndrome in BMS, we have chosen to discuss a possible neurophysiological mechanism based on articles published in international databases conducted by the keywords "pain" and "burning mouth syndrome". Starting from this hypothetical version, this study seeks arguments that the burning mouth syndrome might be due to nonspecific central neuropathic and/ or peripheral disorder. 


\section{Result}

Starting from the concept that pain is a psychological experience that "articulates" around three fundamental components: sensorydiscriminative, motivational and cognitive, it must be admitted that both ordinary tact, pressure, vibration, temperature, and painful sensations as a result of the strong increase in the intensity of sensations of different types would be transmitted encoded into packets of action potentials which, at the cortical levels of the nervous system, are decoded, analyzed and converted into various types of sensations, including those of algebraic type, within a temporal-spatial model [10]. A. Admittance of irrigation deficiency as responsible for neuronal dysfunction should be localized mainly in the lymph nodes in the peripheral cranial nerve tract, leading to sensitivity at the oral/tongue level, which assumes that arteriosclerosis is required to be present in the micro arterioles as their distributors. In these situations, the affected pathways are proto neurons in the case of:

a. Facial, at the level of the ganglion.

b. Glossopharyngeal, Andersch ganglion (sensitivity) and Ehrenritter (general sensitivity).

The pre-angular cranial nerve portion, conventionally referred to as extranevraxial tract, in over $50 \%$ of its thickness contains two types of thermo-algesic sensing conductive fibers:

a. Type A delta $(\mathrm{A} \sigma), 6 \mu \mathrm{m}$ diameter myelin fibers which are usually distributed over the surface, leading to painful inflow at $\geq 10 \mathrm{~m} / \mathrm{s}$.

b. C-type amyelinic fibers, thin, with a diameter of $<2 \mu \mathrm{m}$, which lead slowly, at a speed of $0.5 \mathrm{~m} / \mathrm{s}$, involved in the generation of obtuse, imprecisely localized pain.

Through these nerve fibers, the nerve influx reaches the pericardium of the protoneuron, so that through its axon it propagates to the deuteoneuron of the pathway. This axonal structural portion of the pathway protoneuron is referred to as the conventional intranasal tract. On the trace of this transmission pathway, however, there are interposed between the presinaptic membrane of the protononeuron and the postsynaptic membrane made up of the buttoned terminations of the deuterones dendrites, interstitial neurons with inhibitory function called G cells. The protoneuron-G cell synapse also functions as a "gate", because G cells are synthetic modulators of the natural endogenous analgesia system (secreting enkephaline opioids and especially endorphins). Physiologically, by the action developed by analgetic induction neuromodulators, G cells inhibit the transmission of excitation to the pathway deuteoneuron, even if the receptors/nociceptors were stimulated and, at the level of the first neuron of the pathway, a nervous influx/potential packet action was generated. The action of opioid neuromodulators is the expression of the nervous influx driven by high-conduction A $\sigma$ myelin fibers and is equivalent to "gate closure".
By contrast, the discharging of the nervous influx through the amyelinic fibers of type $\mathrm{C}$ equates to the "gate opening", allowing its synaptic propagation.

The transmission of the nerve influx to a deutoneuron is the expression of the reduction of the discharge frequency of the analgesic neuromodulator by the interstitial neurons.

In BMS, the occurrence of pain in the form of hyperalgesia may be pathogenically explained as expressing the disorder of said physiological mechanism by at least three possibilities, two of which are at the level of the proton of the pathway:

a. either by continuous stimulation of the $\mathrm{C}$ fibers;

b. or by selective reduction of high conductivity fibers, Aб.

\section{Discussion}

The limited duration of the BMS pain with the possibility of returning to periods of time when the pain is absent for it then to reoccur, virtually excludes as a pathogenic mechanism the possibility of the intervention of this second invoked mechanism by quantitatively reducing the number of algo-conductive fibers. This is because the numerical reduction of both $A \sigma$ and selective fibers can only occur through neuronal apoptosis. Since the neuron cannot multiply, at least as the current level of knowledge shows, neuronal apoptosis that cannot allow neuronal regeneration cannot explain the recurrence of the BMS pain after a certain amount of time. Accepting this scientific evidence requires that the "opening of the entrance door" for A $\sigma$ fibers can only occur through qualitative disturbances that disrupt the activity developed by the mediators. Disturbance may be of interest either in the synthesis of neuronal modulators whose prolongations lead at high velocities, or, most likely, the way of the onward flow of neurotransmitters through the protoneuron axon.

Pathogenetically, we admit that all three mechanisms can act synergistically.

A. A possible other pathogenic mechanism may be one in which the excessive synthesis of the neuropeptide $\mathrm{P}$ is involved in the transmission of nociceptive impulse packets. Excessive synthesis occurs at the body and axon level of the first neuron of the thermo-algebraic pathway, generated by variations in neuronal irrigation induced by the ratio of NO and SRO in the perinervascular vascular plexus. In support of these assertions, there are histochemical findings that have highlighted the excessive presence of this substance in the terminal buttons of the axons of the protons of these pathways, whereupon it is released to fix its post membrane specific receptor, neurokinin 1.

B. Morpho-functional lesions through arteriosclerosis may develop beyond the protoneuron headquarters, in the sensorysensitive ascendant tracts involved in the cortical transmission of information collected from the oral mucosa, including lingual. The hypothesis is supported by highlighting the existence of a large 
number of carabinoid receptors at the level of the deutoneurons of the pathway/caudal subunit, which means that the transmission of nociceptive impulses to higher centers is controlled by an endogenous opioid system.

This relatively recent scientific discovery may be an argument in support of our hypothesis that, in order to develop hyperglycemia in BMS, this third pathogenic mechanism, which reduces the synthesis of enkephalins and endorphins, may intervene, but at the level of the $\mathrm{G}$ interneurons, which interpose between proto- and deutoneurons, respectively. The consequence of diminishing opioid synthesis would facilitate neuropeptide $\mathrm{P}$ to develop its nociceptive driving action.

C. Affecting the cortical projection areas of algal, thermal and taste sensitivity as a result of the localization of atherosclerosis in the irrigation vessel wall is a last level that can pathogenically explain the symptoms of BMS. These integrative areas are:

a. For information led by sensory-sensitive fibers of the facial level: areas 3, 1, 2 for sensory impulses, and for those in the areas of ascending parietal circumference.

b. For those submitted by glossopharyngeal level: areas 3,1, 2 and gustatory areas.

c. For the driven nervous influx, related, through the trigeminal fibers: postcentral gyrus.

In support of the pathogenic mechanisms mentioned as responsible for the induction of pain in the primary form of BMS, the results of the studies of Jaaskelainen and Woda which admits that the clinical diagnosis of this idiopathic form of the syndrome comprises at least three distinct, subclinical neuropathic conditions that may overlap in each patient:

a. The first subgroup (50-65\%) is characterized by peripheral neuropathy, which concerns the small diameter fibers of the oral mucosa.

b. The second subgroup (20-25\%) is made up of patients with lingual, mandibular localized pain, or in topographical areas suggesting pathology such as trigeminal neuralgia.

c. And a subgroup (20-40\%) fall within the concept of central pain. The author concedes that pathogenic, the concept of central pain implies the possibility of hypofunction of dopaminergic neurons from the basal ganglia [11].

Scientifically, it is demonstrated that in the processing of information in these areas, an important role is the dopamine neurotransmitter that modulates the control of affectiveemotional states. By synonymy, starting from the observation that in Parkinson's disease due to atherosclerosis the diminution of dopamine concentration was proven, hypothetically, a functional dopaminergic deficiency could be admitted in BMS as well, which would allow the installation of an emotional lability with stressadaptive deficiency [12,13].
The mechanisms through which the co-transducer concentration may decrease may be two:

a. Diminishing of the synthesis into neuroplasm/axoplasm, reducing the number of presynaptic storage vesicles;

b. Interference of $\mathrm{Ca}^{2+}$ influx because change in neurolemia fluidity/permeability disrupts $\mathrm{Ca}^{2+}$ voltage-dependent channel functionality. The consequence would be to reduce the cytoplasmic concentration of $\mathrm{Ca}^{2+}$ that reduces adhesion to presynaptic membrane of dopamine storage vesicles, leading to a diminishing amount of dopamine released into the synaptic gap between sensory-sensitive neurons.

Although action develops in the postsynaptic level in the sympathetic autonomic nervous system, the neuroplastic interference of the $\mathrm{Ca}^{2+}$ influx seems to be potentiated by correlating the action of noradrenaline with ATP release in the extra neuronal environment, which activates some of the specific purine receptors (P2X1) and affects membrane depolarization, sensitizing neuroplastic processes dependent on ion concentration.14 Among other effects developed is the one that alters the vascular reactivity, which acts as a regional oxidative imbalance factor [14].

The scientific acquisitions of the last three decades have argued that through the aging process there is also a double change in the functioning of the structures involved in collecting information and transmitting it as a nervous influx. Thus, beyond the number, distribution, and affinity of receptors, the pathogenic mechanism and the affinity diminishing various components of the information message processing chain, such as guanilatcicylates / G proteins or adenyl cyclase occur. Biochemically, the interaction of the two systems leading to ATP synthesis is demonstrated.

This process, carried out at the neuronal membrane level, synergistically, also involves the energy deficit generated by the release of cytochrome $c$ in the cytosol $[12,15]$. Physiologically, cytochrome $\mathrm{c}$ is located in the mitochondrial intermembrane space, participating in the synthesis of macro-active compounds through the phosphatidyl-inositol chain. The presence of oxidative stress induced by astrocytus-microglia-neuron malization due to atherosclerosis present moves cytochrome c into the cytosol suppressing the functioning of the electron transport chain involved in energetic synthesis.

Moreover, at the neuronal level, the ATP deficit is also accentuated by the intervention of a third pathogenic mechanism: that of the extracellular release of the macroergic compounds. The release of ATP, which is a physiological process, takes place at the level of multiple cell types, the inductive stimulus being mechanical. Its release in pathophysiological conditions explains the appearance of various diseases, including central nervous system dysfunctions.

In support of this pathogenic mechanism, the results of the scientific research from the $21^{\text {st }}$ Century decades show that in general diseases, such as atherosclerosis, hypertension, tissue 
ischemia, and cell lesions, ATP passes from the intracellular to the extracellular environment $[16,17]$.

By accepting for the initiation of pain the hypothesis of the mechanical sensory transduction of the viscera, similarly, in cases of BMS it can be admitted that the release of ATP occurs from the epithelial cells of the oral and lingual mucosa and the attachment would be made on the subtype's purine receptors, P2X3 and P2X2/3 of sensory-free nerve endings, located sub-epithelially. Hence, the nerve propagation propagated as packets of electrical current reaches the sensory protoneuron in the ganglion attached to each of the three cranial nerves involved.

More recent studies acknowledge that after release into the extracellular environment, ATP functions as a pro-nociceptive neurotransmitter/neuromodulator rapidly activating metabotropic $\mathrm{P} 2 \mathrm{Y}$ receptors. Its pro-nociceptive effect is a powerful, long-term action interfering including apoptosis.

Scientific research based on the use of selective antagonists of the first type of purine receptor (P2X receptor) has produced evidence that supports the ability of ATP to initiate and maintain chronic pain after exposure to harmful stimuli as they serve to initiate and sustain the states of increased neuronal excitability. Since the development of the purinergic neurotransmission concept in 1972, numerous scientific evidence has asserted the role of ATP as a synaptic co-transducer only at central level. Recently, numerous experimental research including biochemical measurements, myografies or electrophysiological studies have shown that extracellularised ATP is a major neuromediator at the level of the peripheral nerves as well. This is because P2X receptors that are attached to membrane ion channels play a role in integrating neural and glial cell function into the central nervous system, mediating among other effects chronic neuropathic pain, neuronal degeneration and inflammation [18-20]. The latter may be induced by oxidative stress developed by microangiosclerosis [19].

Such effects as expression of purine receptor activation have as scientific support the ability of the purine to act as a neurotransmitter in modulating vascular reactivity, including cerebral reactivity, apoptosis and secretion of certain cytokines.

\section{Conclusion}

The argument that supports the etiopathogenic hypothesis that a neurodegeneration deficiency would occur in the pathogenesis of BMS is the prevalence of the disease since the sixth decade of life, when arteriosclerosis with the most diverse localization is constituted even if it is not clinically manifested. In the same vein, the most recent results of neurophysiology, psychophysics, neuropathology and imaging research have revealed several neuropathic mechanisms in subclinical stages of the disease suspected at different levels of nevrax. Thus, in patients with BMS, at the sensitive-sensorial cortex, fMRI has been shown to reduce neuronal activation by heat stimuli. On the same note, Jaaskelainen and Woda admits that in the case of pain falling from BMS in the category of cortical pain, pathogenesis would consist of a deficiency of inhibition in the dopaminergic conduction pathways.

Conflicts of Interest: All authors report no conflict of interest.

\section{References}

1. Teruel A, Patel S (2019) Burning mouth syndrome: a review of etiology, diagnosis, and management. Gen Dent 67(2): 24-29.

2. Finnerup NB, Haroutounian S, Kamerman P, Baron R, Bennett DL, et al. (2016) Neuropathic pain: an updated grading system for research and clinical practice. Pain 157(8): 1599-1606.

3. Feller L, Fourie J, Bouckaert M, Khammissa RAG, Ballyram R, et al. (2017) Burning mouth syndrome: Aetiopathogenesis and principles of management. Pain Res Manag 2017: 1926269.

4. Coculescu BI, Manole G, Dincă GV, Dinca AL, Coculescu EC (2019) Considerations on the ethiopathogenesis of algae from the primary form of BMD. Rev Chim (Bucharest) 70(7): 2425-2428.

5. Treede RD, Jensen TS, Campbell JN, Cruccu G, Dostrovsky JO, et al. (2008) Neuropathic pain: redefinition and a grading system for clinical and research purposes. Neurology 70(18): 1630-1635.

6. Vellappally S (2016) Burning mouth syndrome: A review of the etiopathologic factors and management. J Contemp Dent Pract 17(2): 171-176.

7. Hendrix P, Griessenauer CJ, Foreman P, Loukas M, Fisher WS, et al. (2014) Arterial supply of the lower cranial nerves: a comprehensive review. Clinical Anatomy 27(1): 108-117.

8. (2008) Wigley C (section editor) Section 1-Cells, Tissues and Systems. Chapter 3-Nervous system; blood supply of peripheral nerves. In: Standring S, Borley NR, (Eds.), Gray's anatomy: the anatomical basis of clinical practice. ( $40^{\text {th }}$ edn), anniversary edn, Churchill Livingstone: Elsevier, United Kingdom, pp: 41-67.

9. Coculescu BI, Manole G, Coculescu EC, Ionescu E, Popoviciu O, et al. (2018) Autophagy as a neuronal survival mechanism in ischemic stroke. Rom J Leg Med 26(4): 333-339.

10. (2009) Mungiu CO, Cojocaru V (Eds.), Opioid therapy in oncological and non-oncological pain. "Gr. T. Popa" Publishing: University of Medicine and Pharmacy Iaşi, Romania.

11. Jääskeläinen SK, Woda A (2017) Burning mouth syndrome. Cephalalgia 37(7): 627-647.

12. Manole G (2003) General clinical pathophysiology. Vol. 2. The pathophysiology of organs. National Company Printers, Coresi S.A. Publishing: Bucharest, Romania.

13. Coculescu EC, Părlătescu I, Gheorghe C, Țovaru S, Coculescu BI (2017) Management of burning mouth syndrome (BMS) in patients with diabetes mellitus. Rom J Mil Med 120(1): 43-51.

14. Kennedy C (2015) ATP as a cotransmitter in the autonomic nervous system. Auton Neurosci 191: 2-15.

15. Rusu V, Baran T, Brănişteanu DD (1998) Biomembranes and pathology. Medical Publishing House: Bucharest, Romania, Vol.1.

16. Burnstock G (2002) Purinergic signaling and vascular cell proliferation and death. Arterioscler Thromb Vasc Biol 22(3): 364-373. 
17. Lazarowski ER, Boucher RC, Harden TK (2003) Mechanisms of release of nucleotides and integration of their action as $\mathrm{P}_{2} \mathrm{X}$ - and $\mathrm{P}_{2} \mathrm{Y}$-receptor activating molecules. Mol Pharmacol 64(4): 785-795.

18. Jarvis MF (2010) The neural-glial purinergic receptor ensemble in chronic pain states. Trends Neurosci 33(1): 48-57.
19. Ulrich H, Abbracchio MP, Burnstock G (2012) Extrinsic purinergic regulation of neural stem/progenitor cells: implications for CNS development and repair. Stem Cell Rev 8(3): 755-767.

20. Puhakka A, Forssell H, Soinila S, Virtanen A, Röyttä M, et al. (2016) Peripheral nervous system involvement in primary burning mouth syndrome-results of a pilot study. Oral Dis 22(4): 338-344. 